\title{
ON CERTAIN DECOMPOSITIONAL PROPERTIES OF VON NEUMANN ALGEBRAS
}

\author{
by A. B. THAHEEM
}

(Received 21 November, 1985)

1. Introduction. It is well known that if $\alpha$ and $\beta$ are commuting *-automorphisms of a von Neumann algebra $M$ satisfying the equation $\alpha+\alpha^{-1}=\beta+\beta^{-1}$ then $M$ can be decomposed into a direct sum of subalgebras $M p$ and $M(1-p)$ by a central projection $p$ in $M$ such that $\alpha=\beta$ on $M p$ and $\alpha=\beta^{-1}$ on $M(1-p)$ (see, for instance, [6], [7], [2]). Originally this equation arose in the Tomita-Takesaki theory (see, for example, [11]) in the form of one-parameter modular automorphism groups and later on it has been studied for arbitrary automorphisms and one-parameter groups of automorphisms on von Neumann algebras [7], [8], [9]. In the case of automorphism groups satisfying the above equation, one has a similar decomposition but this time without assuming the commutativity condition (cf. [7], [8]). For another relevant work on one-parameter groups of automorphisms which is close to our papers [7] and [8], we refer to Ciorănescu and Zsidó [1]. Regarding applications, this equation has been used for arbitrary automorphisms in the geometric interpretation of the Tomita-Takesaki theory [2] and in the case of automorphism groups it has been a fundamental tool in the generalization of the Tomita-Takesaki theory to Jordan algebras [3]. We may remark that the decomposition in the commuting case [6], [7] is much simpler than in the case of automorphism groups in the non-commutative situation [8]. In some cases one can obtain the decomposition for an arbitrary pair of automorphisms without assuming their commutativity but the problem in the general case has been unresolved. Recently we have shown that if $\alpha$ and $\beta$ are *automorphisms of a von Neumann algebra $M$ satisfying the equation $\alpha+\alpha^{-1}=\beta+\beta^{-1}$ (without assuming the commutativity of $\alpha$ and $\beta$ ) then there exists a central projection $p$ in $M$ such that $\alpha^{2}=\beta^{2}$ on $M p$ and $\alpha^{2}=\beta^{-2}$ on $M(1-p)[10]$.

The main purpose of this note is to show that, in this situation, the von Neumann algebra $M$ can, in fact, be decomposed into three parts such that $\alpha=\beta$ on the first part, $\alpha=\beta^{-1}$ on the second part and $\alpha^{4}=\beta^{4}=1$ on the third part. This provides a better understood decomposition of $M$ as a solution of the above equation than we have obtained so far in [5], [6] and [7]. We close the paper with an interesting result about the residual spectrum of automorphisms of von Neumann algebras which is of independent interest.

We gratefully acknowledge many useful suggestions by Professor A. van Daele during this research.

2. Decomposition results. The following result is partly known (see, for instance, [6]). However, we include it here giving a variant and a simpler proof.

Proposition 2.1. Let $\alpha$ be $a^{*}$-automorphism of a von Neumann algebra $M$. Then the

Glasgow Math. J. 29 (1987) 177-179. 
weak closure of the algebra generated by $R(\alpha-1)$ (the range of $(\alpha-1)$ ) is a two-sided ideal in $M$ and the orthogonal ideal is contained in $N(\alpha-1)$ (the null space of $(\alpha-1)$ ).

Proof. Let $L$ be the $\sigma$-weak closure of the algebra generated by $R(\alpha-1)$. If $x \in N(\alpha-1)$ and $y \in M$ then $x(\alpha-1)(y)=x \alpha(y)-x y=\alpha(x y)-x y=(\alpha-1)(x y)$. Hence $N(\alpha-1) \cdot R(\alpha-1) \subseteq R(\alpha-1)$. Similarly we have that $R(\alpha-1) . N(\alpha-1) \subseteq$ $R(\alpha-1)$. The fact that $R(\alpha-1)+N(\alpha-1)$ is $\sigma$ - weakly dense in $M$ [5] implies that $L$ is a two-sided ideal in $M$. Therefore $L=M p$ for a central projection $p$ in $M$. Let $L^{\perp}=M(1-p)$ be the orthogonal ideal. We shall prove that $L^{\perp} \subseteq N(\alpha-1)$.

Clearly $p$ is $\alpha$-invariant because $\alpha R(\alpha-1) \subseteq R(\alpha-1)$. Let $\alpha^{\prime}$ be the restriction of $\alpha$ on $M(1-p)$. Again $N\left(\alpha^{\prime}-1\right)+R\left(\alpha^{\prime}-1\right)$ is dense in $M(1-p)$. Since $R\left(\alpha^{\prime}-1\right) \subseteq$ $R(\alpha-1) \subseteq M p$, therefore $R\left(\alpha^{\prime}-1\right)=\{0\}$ which implies that $N\left(\alpha^{\prime}-1\right)$ is dense in $M(1-p)$. Thus $N(\alpha-1) \supseteq N\left(\alpha^{\prime}-1\right)=M(1-p)$. This proves the proposition.

THEOREM 2.2. Let $\alpha$ and $\beta$ be ${ }^{*}$-automorphisms of a von Neumann algebra $M$ such that $\alpha+\alpha^{-1}=\beta+\beta^{-1}$. Then $M$ can be decomposed into three parts such that

(i) $\alpha=\beta$ on the first part,

(ii) $\alpha=\beta^{-1}$ on the second part,

(iii) $\alpha^{4}=\beta^{4}=1$ on the third part.

Proof. It follows from the main decomposition theorem of [10] that $\alpha^{2}=\beta^{2}$ on $M p$ and $\alpha^{2}=\beta^{-2}$ on $M(1-p)$. First assume that $\alpha^{2}=\beta^{2}$. Then $\alpha+\alpha^{-1}=\beta+\beta^{-1}$ and $\alpha^{2}=\beta^{2}$ imply that

$$
\alpha^{-1}\left(\alpha^{2}+1\right)=\beta^{-1}\left(\beta^{2}+1\right)=\beta^{-1}\left(\alpha^{2}+1\right) .
$$

So $\alpha=\beta$ on $R\left(\alpha^{2}+1\right)$. Now

$$
R\left(\alpha^{4}-1\right)=R\left(\left(\alpha^{2}+1\right)\left(\alpha^{2}-1\right)\right) \subseteq R\left(\alpha^{2}+1\right)
$$

and by the density of $R\left(\alpha^{4}-1\right)+N\left(\alpha^{4}-1\right)$ it follows that $R\left(\alpha^{2}+1\right)+N\left(\alpha^{4}-1\right)$ is dense. By Proposition 2.1 above, the algebra generated by $R\left(\alpha^{4}-1\right)$ (and hence the algebra generated by $R\left(\alpha^{2}+1\right)$ ) is a two-sided ideal given by $q M$ (where $q$ is a central projection in $M$ ) and the orthogonal ideal is contained in $N\left(\alpha^{4}-1\right)$. Therefore we obtain that $\alpha=\beta$ on $M q$ and $\alpha^{4}=1$ (and $\alpha^{2}=\beta^{2}$ ) on $M(1-q)$ or equivalently $\alpha^{4}=\beta^{4}=1$ on $M(1-q)$.

Similarly when we consider the part $M(1-p)$, we get that $\alpha=\beta^{-1}$ on a part of $M$ and $\alpha^{4}=\beta^{4}=1$ on the other part. So in any case we get three parts of $M$ with the required properties.

We conclude the paper with an interesting result about the spectrum of *automorphisms of von Neumann algebras. It is well known that a normal operator on a Hilbert space has empty residual spectrum (see, for instance, [4, p. 325]). we prove a similar result for ${ }^{*}$-automorphisms of von Neumann algebras. This result may be known but we have not seen it in the literature. So we include it here.

Proposition 2.3. The residual spectrum of $a^{*}$-automorphism of a von Neumann algebra is empty. 
Proof. Let $\alpha$ be a ${ }^{*}$-automorphism of a von Neumann algebra $M$. Suppose that $\lambda$ is in the residual spectrum of $\alpha$. Then $N(\alpha-\lambda)=\{0\}$ and $R(\alpha-\lambda)$ is not $\sigma$-weakly dense in $M$. There exists a non-zero self-adjoint continuous linear functional $\phi$ in $M_{*}$ such that $\phi((\alpha-\lambda)(x))=0$ for all $x$ in $M$. Let $\phi=|\phi| \circ u$ be the polar decomposition of $\phi \circ \alpha$. It follows that $\phi=(|\phi| \circ \alpha) \circ \bar{\lambda} \alpha^{-1}(u)$ is again the polar decomposition of $\phi$. Therefore $|\phi|$ is $\alpha$-invariant and hence by the uniqueness of the polar decomposition, $u=\bar{\lambda} \alpha^{-1}(u)$ or $\alpha(u)=\bar{\lambda} u$. This shows that $\alpha\left(u^{*}\right)=\lambda\left(u^{*}\right)$. Then either $\lambda \in \sigma_{p}(\alpha)$ or $u=0$. Thus $\phi=0$, a contradiction. This completes the proof of the proposition.

\section{REFERENCES}

1. I. Ciorănescu and L. Zsido, Analytic generators for one-parameter groups, Tôhoku Math. J. (2) 28 (1976), 327-362.

2. U. Haagerup and C. F. Skau, Geometric aspects of the Tomita-Takesaki theory II, Math. Scand. 48 (1981), 241-252.

3. U. Haagerup and $\mathrm{H}$. Hanche-Olsen, Tomita-Takesaki theory for Jordan algebras, $J$. Operator Theory 11 (1984), 343-364.

4. W. Rudin, Functional analysis (Tata McGraw-Hill, 1974).

5. A. B. Thaheem, Decomposition of a von Neumann algebra relative to a *-automorphism, Proc. Edinburgh Math. Soc. (2) 22 (1979), 9-10.

6. A. B. Thaheem, Decomposition of a von Neumann algebra, Rend. Sem. Mat. Univ. Padova 65 (1981), 1-7.

7. A. B. Thaheem and M. Awami, A short proof of a decomposition theorem of a von Neumann algebra, Proc. Amer. Math. Soc. 92 (1984), 81-82.

8. A. B. Thaheem, A. van Daele and L. Vanheeswijck, A result on two one-parameter automorphism groups, Math. Scand. 51 (1982), 261-274.

9. A. B. Thaheem, A bounded map associated to a one-parameter group of *-automorphisms of a von Neumann algebra, Glasgow Math. J. 25 (1984), 135-140.

10. A. B. Thaheem, On pairs of automorphisms of von Neumann algebras, preprint.

11. A. van Daele, A new approach to the Tomita-Takesaki theory of generalized Hilbert algebras, J. Funct. Anal. 15 (1974), 387-393.

Department of Mathematics

QUAID-I-AZAM UNIVERSITY

IsLAMABAD, PAKISTAN 